\title{
Content validity and psychometric evaluation of the Functional Assessment of Chronic Illness Therapy-Fatigue scale in patients with chronic lymphocytic leukemia
}

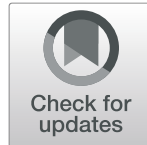

Daniel Eek ${ }^{1 *}$, Cristina Ivanescu ${ }^{2}$, Laura Corredoira ${ }^{3}$, Oren Meyers ${ }^{4}$ and David Cella ${ }^{5}$

\begin{abstract}
Purpose: Fatigue is a prominent symptom in individuals with chronic lymphocytic leukemia (CLL). This work evaluates the content validity and psychometric properties of the Functional Assessment of Chronic Illness TherapyFatigue scale (FACIT-Fatigue) in patients with CLL to determine if it is fit for purpose in CLL research.

Methods: The FACIT-Fatigue yields a 13-item total score from a five-item symptom subscale and an eight-item impact subscale. To evaluate content validity, cognitive debriefing interviews were conducted with 40 patients with $\mathrm{CLL}$ in the first-line or relapsed or refractory setting. Psychometric properties, including structural validity, internal consistency, construct and known-groups validity, were investigated using data from a phase 3 trial in relapsed or refractory CLL (NCT02970318).

Results: Interviewed patients considered the FACIT-Fatigue items relevant to their CLL experience, understood the terminology and agreed with response options. Confirmatory factor analysis confirmed the presence of symptom and impact subscales, but also supported unidimensionality of the FACIT-Fatigue. The FACIT-Fatigue total, symptom and impact subscales demonstrated good internal consistency (Cronbach's coefficient $a>0.85$ and McDonald's omega $\omega>0.90$ ), and strong correlations with relevant EORTC QLQ-C30 scales (all Spearman's $r \geq 0.5$ ). Known-groups validity was shown by significant differences between groups defined by baseline performance status, hemoglobin level and constitutional symptoms (all $p<.0001$ ). Cluster analysis supported FACIT-Fatigue score thresholds of 30 and 34 to define a severe fatigue population.
\end{abstract}

Conclusions: Content validity and psychometric evaluation in patients with CLL demonstrated that the FACIT-Fatigue has good psychometric properties and is fit for purpose in CLL.

Keywords: Chronic lymphocytic leukemia, Content validity, FACIT-Fatigue, Psychometric properties

\footnotetext{
* Correspondence: daniel.eek@astrazeneca.com

${ }^{1}$ AstraZeneca Gothenburg, Pepparedsleden 1, 43150 Mölndal, Sweden

Full list of author information is available at the end of the article
}

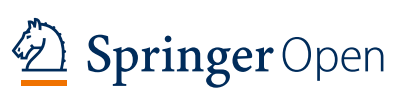

(c) The Author(s). 2021 Open Access This article is licensed under a Creative Commons Attribution 4.0 International License, which permits use, sharing, adaptation, distribution and reproduction in any medium or format, as long as you give appropriate credit to the original author(s) and the source, provide a link to the Creative Commons licence, and indicate if changes were made. The images or other third party material in this article are included in the article's Creative Commons licence, unless indicated otherwise in a credit line to the material. If material is not included in the article's Creative Commons licence and your intended use is not permitted by statutory regulation or exceeds the permitted use, you will need to obtain permission directly from the copyright holder. To view a copy of this licence, visit http://creativecommons.org/licenses/by/4.0/. 


\section{Introduction}

Chronic lymphocytic leukemia (CLL) is a long-term disease that typically develops and progresses slowly. In patients with CLL, abnormal lymphocytes accumulate in the blood, bone marrow and lymphatic tissues over time, resulting in anemia, bleeding and increased susceptibility to infections [1, 2]. Fatigue is one of the main symptoms of hematological cancers such as CLL, and is thought to be caused by underlying anemia and pathophysiological pro-inflammatory disease mechanisms [3]. In patients with cancer, moderate to severe anemia (hemoglobin [Hb] levels $<110$ $\mathrm{g} / \mathrm{L}$ [4]) has been shown to be associated with persistent fatigue and generalized weakness [3], and anemia severity correlates with the degree of fatigue [5].

In a recent qualitative interview study, patients with CLL reported fatigue as a key symptom and impact of their disease and its treatment [6]. The manifestations of fatigue included a lack of energy, weakness, decreased physical functioning and a reduced ability to maintain professional and social roles [6]. The importance of fatigue as a central symptom and impact of CLL, and as a potential indicator of disease severity, makes it relevant to measure in clinical trials assessing new treatments for CLL. The Functional Assessment of Chronic Illness Therapy-Fatigue scale (FACIT-Fatigue) is a patientreported outcome (PRO) instrument designed to assess fatigue-related symptoms and their impact on daily functioning. The FACIT-Fatigue comprises a five-item symptom subscale and an eight-item impact subscale; in total, the scale includes 13-items. Previous work in general and cancer population samples indicates that the fatigue experience and the impact of fatigue are aligned on one single dimension and supports the FACIT-Fatigue total score as a reasonable endpoint choice for clinical research [7]. However, factor analysis has also indicated that the two subscales can be employed and reported separately, should this be needed in specific settings [7]. To be fit for purpose, PRO instruments need to have documented reliability and validity in the intended target patient population [5]. The FACIT-Fatigue has extensive published evidence of its reliability and validity in patients with cancer [7-11], although not specifically with CLL.

The current study evaluates the content validity and psychometric properties of the FACIT-Fatigue in patients with CLL to determine if it is fit for purpose in this population.

\section{Methods}

Content validity of the FACIT-Fatigue was evaluated in qualitative interviews with CLL in the first-line $(1 \mathrm{~L})$ setting or in the relapsed or refractory $(R / R)$ setting. The interviews included cognitive debriefing of the instrument. Reliability and validity of the FACIT-Fatigue were assessed in patients with R/R CLL enrolled in a phase 3 trial assessing acalabrutinib in $\mathrm{R} / \mathrm{R}$ CLL (ASCEND; NCT02970318) [12].

\section{Measures \\ FACIT-fatigue}

The FACIT-Fatigue includes a five-item symptom subscale and an eight-item impact subscale that together make up the 13-item total score. Item responses range from 0 ('not at all') to 4 ('very much'). Scores for negatively worded items are reversed, such that higher scores are better (i.e. less fatigue). The FACIT-Fatigue total score ranges from 0 to 52 (the general population mean score is $43[5,13])$. The recall period for each item is the past 7 days.

\section{EORTC QLQ-C30}

The European Organisation for Research and Treatment of Cancer Quality of Life Questionnaire-Core 30-questions (EORTC QLQ-C30) contains five multiitem function scales (physical, role, cognitive, emotional, social), three symptom scales (fatigue, pain, nausea/vomiting), five single-item symptoms (dyspnea, insomnia, appetite loss, constipation, diarrhea), a global health status scale and a single-item financial impact question. High function scale or global health status scale scores represent a high level of functioning and a high quality of life, respectively, whereas a high symptom score 'represent' a high level of symptomatology/problems.

\section{EQ-5D-5L and EQ-VAS}

The 5-level, 5-dimension EuroQol questionnaire (EQ5D-5L) comprises five impairment-related dimensions (mobility, self-care, usual activities, pain/discomfort, anxiety/depression). Each dimension is defined from 1, indicating no problem, to 5 , indicating extreme problems. Its global health visual analogue scale (EQ-VAS) is a $0-100$ scale of a patient's health status, where 0 represents the 'worst health you can imagine' and 100 the 'best health you can imagine'.

\section{Cognitive debriefing interviews}

As part of a qualitative interview study [6], cognitive debriefing interviews were conducted with 40 patients with $1 \mathrm{~L}$ CLL or R/R CLL resident in the United States. Full methods and results of the concept elicitation part of the interview study have been published previously [6] Potential participants were identified via a patient advocacy organization (CLL Society; https://cllsociety.org) and two market research firms (Liberating Research, www. liberatingresearch.com; and Rare Patient Voice, https:// rarepatientvoice.com), and were contacted by email and telephone about study details and participation. To be 
eligible, patients needed to be aged 18 years or older, be diagnosed with CLL, have a self-reported Eastern Cooperative Oncology Group (ECOG) Performance Status score $\leq 2$, be proficient in English and have experienced at least one constitutional symptom of CLL (fatigue, weight loss, fever or night sweats) in the past week. Patients in the R/R CLL group had to have received two or more lines of treatment specifically to treat CLL.

The qualitative interviews were carried out by telephone and generally lasted $60-75 \mathrm{~min}$ in total for the concept elicitation and cognitive debriefing parts combined. Interviews were conducted by trained interviewers (O. Meyers, C. Krogh, S. Lee; IQVIA). Patients completed the FACIT-Fatigue as part of cognitive debriefing. During cognitive debriefing, participants were asked to review the FACIT-Fatigue. Patients' observations of the FACIT-Fatigue were grouped by feedback on the instrument instructions (clarity, difficulty understanding), individual items, response options and the questionnaire as a whole (missing and redundant items).

De-identified transcripts of patient interviews were coded using ATLAS.ti software (version 8). Two coders, who had also moderated most of the patient interviews, coded the results of and feedback on the FACIT-Fatigue. Inter-coder agreement was assessed periodically throughout the coding process, and any disagreement was discussed and addressed.

\section{Psychometric analysis in CLL}

Data for the psychometric analysis of the FACIT-Fatigue were from baseline assessments in the phase 3 ASCEND trial (NCT02970318), a multicenter, openlabel study that enrolled patients with R/R CLL [12]. Eligible patients were aged 18 years or older, had previously been treated with at least one systemic therapy and had an ECOG Performance Status score $\leq 2$. Patients were randomized 1:1 to acalabrutinib $100 \mathrm{mg}$ twice daily or investigator's choice of therapy (either idelalisib $150 \mathrm{mg}$ twice daily plus rituximab $[375 \mathrm{mg} /$ $\mathrm{m}^{2}$ intravenously on day 1 of cycle 1 , then $500 \mathrm{mg} / \mathrm{m}^{2}$ intravenously every 2 weeks for 4 doses and thereafter every 4 weeks for 3 doses] or bendamustine $70 \mathrm{mg} / \mathrm{m}^{2}$ intravenously on day 1 and 2 of each 28-daycycle plus rituximab $\left[375 \mathrm{mg} / \mathrm{m}^{2}\right.$ intravenously on day 1 of cycle 1 , then $500 \mathrm{mg} / \mathrm{m}^{2}$ intravenously on day 1 of cycles 2-6]).

Patients in the ASCEND trial completed the FACIT-Fatigue, EORTC QLQ-C30, EQ-5D-5L and EQ-VAS at baseline and during the study. Mean total, subscale and item scores were calculated. The presence of floor effects $(>25 \%$ of patients scoring 'worst possible health state') and ceiling effects ( $>25 \%$ of patients scoring 'best possible health state') was assessed.

\section{Confirmatory factor analysis}

Confirmatory factor analysis was employed to evaluate the latent structure (i.e. underlying subscales) of the FACIT-Fatigue instrument. First, a single factor model of the FACIT-Fatigue was examined to determine the unidimensionality of all 13 items of the instrument. If the model fits the data well and all item factor loadings are greater than 0.3, the FACIT-Fatigue can be considered unidimensional [7]. Next, a bifactor model was examined [14-16]. The bifactor model comprised a general factor of all 13 items, and two sub-domain factors, which were defined by the five symptom items and eight impact items, respectively. If all general factor item loadings are greater than 0.3 and loadings are higher on the general factor than they are on the sub-domains, the general factor can then be considered measurable even in the presence of sub-domain factors [7].

Unidimensionality was evaluated by examining fit statistics of the confirmatory factor analysis (CFA) models and the investigation of factor loadings to assess the relative impact of the secondary dimensions. The following fit indices were evaluated: the root mean square error of approximation (RMSEA) [17]; standardized root mean square residual (SRMR) [18]; comparative fit index (CFI) [19]. The RMSEA and SRMR measure the discrepancy between the observed sample and the hypothesized model. The CFI is an incremental fit index with the null hypothesis that all components in the model are uncorrelated. In addition, factor loadings from single factor modeling and loadings on the general factor of the bifactorial model were compared to assess the level of disturbance due to multidimensionality in the data [20]. Standard cutoff values were used for RMSEA $(<0.06)$, SRMR $(<0.08)$ and $(C F I>0.95)[17-20]$.

Model identification was ensured by restricting the factor variance to 1 , making sure that at least three indicator variables per latent factor were considered and verifying that the number of datapoints was larger than the number of parameters to be estimated. Mplus 8.0 was used to perform the factor analyses. We employed the mean and variance-adjusted weighted least-squares (WLSMV) estimator, suitable for the analysis of categorical data, polychoric correlations and theta parameterization - in which residual variances of observed categorical outcome variables are allowed to be parameters in the models [21]. A pairwise present approach to missing data was used as it is the default in Mplus with WLSMV estimator [22].

\section{Internal consistency reliability}

Internal consistency reliability is a measure that summarizes the correlations across instrument items. Cronbach's coefficient $\alpha$ was used to assess internal consistency reliability of the FACIT-Fatigue symptom 
subscale, the impact subscale and the total scale. A Cronbach's coefficient $\alpha \geq 0.70$ indicates acceptable reliability [23]. In addition, McDonald's omega $(\omega)$ and omega hierarchical $(\omega \mathrm{H})$ coefficients were calculated as they provide better estimates of measurement precision (reliability) than the traditional Cronbach's alpha [24]. Omega coefficients estimate the proportion of variance in unit-weighted total score attributable to all sources of common variance and to the general factor within the bifactor framework $[16,25,26]$. A high $\omega$ value suggests a highly reliable multidimensional composite and a high $\omega \mathrm{H}$ value $(>0.80)$, when a bifactor structure is employed, suggests that the general factor is the dominant source of systematic variance with sub-domain factors having less influence. The unidimensionality of the index was also evaluated by calculating the Explained Common Variance index (ECV) $[27,28]$. Higher values of ECV indicate a strong general factor allowing us to fit a unidimensional model even to multidimensional data.

\section{Construct validity}

Construct validity examines the relationship among scales that measure similar concepts (convergent validity) and among scales that measure different concepts (divergent validity). Convergent validity and divergent validity were assessed to explore associations between the FACIT-Fatigue and the EORTC QLQ-C30 and EQVAS, using Spearman's rank correlation coefficients. Spearman's rank correlation coefficients $\geq 0.50$ were considered to demonstrate convergent validity; Spearman's rank correlation coefficients $<0.30$ demonstrated divergent validity [29]. Moderate to high correlations were expected between the FACIT-Fatigue scores and the fatigue scale from the EORTC QLQ-C30, supporting convergent validity. Although fatigue is likely to affect most aspects of quality of life, low correlations were expected between the FACIT-Fatigue scores and gastrointestinal-related scales (e.g. constipation) from the EORTC QLQ-C30, supporting divergent validity.

\section{Known-groups validity}

Known-groups validity is a form of construct validity that explores if scales differentiate between groups that are hypothesized a priori to differ. FACIT-Fatigue scores between groups known to be different were compared using analysis of variance (ANOVA) on baseline data. Known groups comparisons were explored based on ECOG Performance Status score (0 [fully active] vs 1 or 2 [restricted activity but still ambulatory and capable of all selfcare]), Hb level ( $\geq 110 \mathrm{~g} / \mathrm{L}$ [no/mild anemia] vs $<$ $110 \mathrm{~g} / \mathrm{L}$ [moderate/severe anemia] [4]) and constitutional symptoms (night sweats, fever, unexplained weight loss, significant fatigue [none vs $\geq 1$ symptom]). It was hypothesized that patients with an ECOG score $\geq 1$, with moderate to severe anemia or with constitutional symptoms would have lower FACIT-Fatigue scores (more fatigue) than patients with an ECOG score of 0 , no moderate to severe anemia or with no constitutional symptoms. The following baseline covariates were included: sex (male vs female) and age. Because so few patients had an ECOG Performance Status score of 2, the known groups employed here differ from the stratification of 0 or 1 vs 2 which was used as part of the stratified randomization in the ASCEND trial.

\section{Defining the severity cut-off score}

Severity cut-off scores were explored for differentiating between patients with low symptom levels and those with higher symptom levels, to define a severe fatigue population. Cluster analysis was performed to identify a FACIT-Fatigue severity cut-off score [30]. Clusters were formed using the FACIT-Fatigue symptom subscale and EORTC QLQ-C30 Fatigue scale scores on one hand, and using individual FACIT-Fatigue and EORTC QLQC30 item scores on the other hand. Scores were first standardized on their ranges to equalize the influence of variables with different scale lengths on the cluster solution. A two-step cluster analysis using SPSS [31] was then used to determine the cluster membership. An analysis by Cella et al. suggested one standard deviation (SD) below the general population mean of 43 (SD: 9) to denote the threshold for fatigue impairment, resulting in a cut-off value of 34 [5]. In addition, a FACIT-Fatigue threshold of 30 for fatigue impairment, suggested by Piper and Cella [32], was also considered in our analysis. Agreement between the clusters and thresholds was assessed using Cohen's kappa coefficient [33]. Cohen characterized values $\leq 0$ as indicating no agreement, and $0.01-0.20$ as slight, $0.21-0.40$ as fair, $0.41-0.60$ as moderate, $0.61-0.80$ as substantial and $0.81-1.00$ as almost perfect agreement.

\section{Results \\ Cognitive debriefing interviews}

The interview study included 20 patients with $1 \mathrm{~L}$ CLL and 20 patients with R/R CLL. Median age of participants was 58 (range: 28-73) years, and sex distribution was approximately equal (men: $52 \%$; women: $48 \%$ ). The mean FACIT-Fatigue score among the patients participating in the qualitative interview study was 28.9 (SD: 13.6) for patients with $1 \mathrm{~L}$ CLL and 29.3 (SD: 11.5) for those with R/R CLL, out of a possible maximum score of 52. All patients confirmed that the FACIT-Fatigue was reflective of their experiences with CLL-related fatigue. Patients confirmed that most of the terminology was clear and well understood, and that the wording frequently reflected that used by patients with CLL-related 
Table 1 Selection of feedback on the FACIT-Fatigue from patients with CLL

\begin{tabular}{|c|c|}
\hline $\begin{array}{l}\text { Characteristics of } \\
\text { FACIT-Fatigue }\end{array}$ & Feedback from patients \\
\hline $\begin{array}{l}\text { Clarity of instructions and } \\
\text { items }\end{array}$ & $\begin{array}{l}\text { "I think they're clear and I think that they're valid." (Patient with } 1 \text { L CLL) } \\
\text { "Straightforward, and easy, and clear." (Patient with } 1 \text { L CLL) } \\
\text { "I think these questions are all pretty well designed." (Patient with R/R CLL) }\end{array}$ \\
\hline $\begin{array}{l}\text { Suitability of response } \\
\text { options }\end{array}$ & $\begin{array}{l}\text { "There are enough options. It is better than saying yes or no." } \\
\text { "I think [the distinctions between } 0 \text { and 4] are pretty clear." }\end{array}$ \\
\hline $\begin{array}{l}\text { Relevance to patients } \\
\text { with CLL }\end{array}$ & $\begin{array}{l}\text { Overall } \\
\text { "I think it's pretty thorough. It states everything from activities to the energy to the eating, a lot of things that I know I've } \\
\text { struggled with." (Patient with } 1 \mathrm{LCLL} \text { ) } \\
\text { "I think these questions are spot on [in making people understand my fatigue]." (Patient with } 1 \mathrm{LCLL} \text { ) } \\
\text { Item } 10 \text { ("I am too tired to eat") } \\
\text { "I can't imagine anybody being too tired to lift a fork, but I feel if you're too tired to eat, it's probably because you're physically } \\
\text { exhausted, and there is some effort involved in making or getting food for yourself that you just don't want to put the effort } \\
\text { in." (Patient with R/R CLL) } \\
\text { "That's very clear and probably very pertinent for somebody who's really that tired. I'm sure it happens." (Patient with R/R CLL) } \\
\text { Item } 3 \text { ("I feel listless/washed out") } \\
\text { "This one was a little unclear. Washed out is not a term that I use or I'm familiar with. When I think of listless, I think of need } \\
\text { something to do. Almost bored or fidgety." (Patient with } 1 \mathrm{LCLL)}\end{array}$ \\
\hline
\end{tabular}

Abbreviations: CLL chronic lymphocytic leukemia, FACIT-Fatigue Functional Assessment of Chronic Illness Therapy-Fatigue scale, $1 L$ first-line setting, $R / R$ relapsed or refractory setting

fatigue. Table 1 shows a selection of patients' feedback on the FACIT-Fatigue.

Patients considered the FACIT-Fatigue items to be relevant to patients with CLL and distinct from each other, although the item 'I am too tired to eat' was not considered to be highly pertinent. Respondents could imagine that some patients with CLL might be too tired to eat, but they recalled being too tired to prepare a meal, rather than too tired to eat it. However, respondents felt that the item was still relevant and that it did not detract from the applicability or clarity of the FACIT-Fatigue. Fatigue-related impact items were thought to capture adequately both the mental and physical impacts of patients' fatigue.

The terminology of the FACIT-Fatigue was found to be clear, except for the item 'I feel listless/washed out' (item 3), which patients did not consistently understand as intended. Most patients linked 'I feel listless/washed out' to an absence of both physical and mental energy, but some interpreted it just as a lack of physical energy.

All patients found the response options provided by the FACIT-Fatigue to be suitable and sufficient. Conceptual relevance of the FACIT-Fatigue was supported by mapping of its items to the seven fatigue-related sub-components identified during concept elicitation [6] (Table 2).

\section{Psychometric analysis in CLL}

Baseline PRO data were available for 263 patients (85\%) enrolled in the phase 3 ASCEND trial. Median age was 67 (range: $32-89$ ) years and 67\% were men. At baseline, 231 patients $(88 \%)$ had an ECOG Performance Status score of 0 or 1 , and 32 patients (12\%) had an ECOG Performance Status score of 2. The baseline mean FACITFatigue score was 35.27 (SD: 9.87) for the total scale, out of a possible maximum score of 52, and was 12.09 (SD: 4.34) and 23.18 (SD: 6.12) for the symptom and impact subscale, respectively. Mean FACIT-Fatigue scores ranged from 1.01 to 1.84 for symptom items and from 0.40 to 2.23 for impact items. Some ceiling effects (scoring 'best possible health state') were observed: the proportion of patients who answered 'not at all' (indicating that they did not have the symptom or impact) was above $25 \%$ for only one of the five symptom subscale items (item 3, 'I feel listless/washed out'); however, more than $25 \%$ of patients answered 'not at all' for seven of the eight impact subscale items, indicating that there was low impact of fatigue on activities at baseline (Supplemental File 1). No floor effects (scoring 'worst possible health state') were observed (Supplemental File 1).

\section{Confirmatory factor analysis}

The one-factor solution (Table 3) showed acceptable fit based on the CFI (0.946), SRMR (0.066) and the loadings (all factor loadings $>0.30$ ) and a poor fit based on the RMSEA (0.152; 95\% confidence interval [CI]: 0.1390.165). The individual subscales also showed good fit when examining the CFI and the loadings and a poor fit when examining the RMSEA. The bifactor model showed acceptable fit based on the CFI (0.973), SRMR (0.044) and a poor fit based on the RMSEA (0.120; $95 \%$ CI: 0.105-0.135).

All factor loadings were statistically significant for the one factor (Supplemental File 2). For the bifactor model all factors were significant for the general factor, but for the sub-domain factors item 'I have energy' (item 7) loading on the fatigue symptom domain and item 'I need to sleep during the day' (item 9) on the fatigue impact domain were not significant (Supplemental File 3). 
Table 2 Mapping FACIT-Fatigue items to fatigue-related sub-components identified during concept elicitation

\begin{tabular}{|c|c|c|c|c|c|c|c|}
\hline \multirow[b]{2}{*}{ FACIT-Fatigue item } & \multicolumn{4}{|c|}{ Symptom concepts } & \multicolumn{3}{|l|}{ Impact concepts } \\
\hline & $\begin{array}{l}\text { Tiredness/ } \\
\text { need for } \\
\text { sleep }\end{array}$ & $\begin{array}{l}\text { Lack of } \\
\text { energy }\end{array}$ & Weakness & $\begin{array}{l}\text { Cognitive } \\
\text { fatigue }\end{array}$ & $\begin{array}{l}\text { Decreased ability to maintain } \\
\text { social/familial/professional } \\
\text { role }\end{array}$ & $\begin{array}{l}\text { Decreased } \\
\text { physical } \\
\text { functioning }\end{array}$ & Frustration \\
\hline 1. I feel fatigued & $\checkmark$ & $\checkmark$ & $\checkmark$ & $\checkmark$ & & & \\
\hline 2. I feel weak all over & & & $\checkmark$ & & & & \\
\hline 3. I feel listless/washed out & & $\checkmark$ & & $\checkmark$ & & & \\
\hline 4. I feel tired & $\checkmark$ & & & & & & \\
\hline $\begin{array}{l}\text { 5. I have trouble starting things } \\
\text { because I am tired }\end{array}$ & & $\checkmark$ & & $\checkmark$ & $\checkmark$ & $\checkmark$ & \\
\hline $\begin{array}{l}\text { 6. I have trouble finishing things } \\
\text { because I am tired }\end{array}$ & & $\checkmark$ & & $\checkmark$ & $\checkmark$ & $\checkmark$ & \\
\hline 7. I have energy & & $\checkmark$ & & & & & \\
\hline $\begin{array}{l}8.1 \text { am able to do my usual } \\
\text { activities }\end{array}$ & & & & & $\checkmark$ & $\checkmark$ & \\
\hline 9. I need to sleep during the day & $\checkmark$ & & & & & & \\
\hline 10. I am too tired to eat & & & & & & $\checkmark$ & \\
\hline $\begin{array}{l}11 . \text { I need help doing my usual } \\
\text { activities }\end{array}$ & & & & & & $\checkmark$ & \\
\hline $\begin{array}{l}\text { 12. I am frustrated by being too } \\
\text { tired to do the things I want to } \\
\text { do }\end{array}$ & & & & & & & $\checkmark$ \\
\hline $\begin{array}{l}\text { 13. I have to limit my social } \\
\text { activity because I am tired }\end{array}$ & & & & & $\checkmark$ & & \\
\hline
\end{tabular}

Abbreviation: FACIT-Fatigue Functional Assessment of Chronic Illness Therapy-Fatigue scale

Results from the bifactor model showed that almost all items had higher loadings on the general factor (range: 0.43 to 0.95 ) than on the two sub-domain factors (symptoms, range: -0.06 to 0.47 ; impacts, range: -0.21 to 0.66), supporting the essential unidimensionality of the FACIT-Fatigue. In addition, loadings on the general factor were very similar to the factor loadings of the single factor analysis.

\section{Internal consistency reliability}

Cronbach's coefficient $\alpha$ was 0.87 (95\% CI: 0.84-0.89) for the FACIT-Fatigue symptom subscale, 0.86 (95\% CI: $0.83-0.88$ ) for the impact subscale and 0.91 (95\% CI: $0.90-0.93)$ for the total scale. McDonald's $\omega$ was 0.94 for the FACIT-Fatigue total scale, 0.91 for the impact subscale and 0.90 for the symptom subscale. For the bifactor model $\omega$ coefficient was 0.95 and $\omega \mathrm{H}$ was 0.91 . A comparison of $\omega \mathrm{H}(0.91)$ with $\omega(0.95)$ is critical. Here, we see that almost all of the reliable variance in total scores $(0.91 / 0.95=0.96)$ can be attributed to the general factor, assumed to reflect individual differences on the trait of fatigue. Only $4 \%$ ( $\omega$ $\omega \mathrm{H})$ of the reliable variance in total scores can be attributed to the multidimensionality caused by the subgroup factors. ECV was 0.82, also indicating a quite strong general factor accounting for well over half the common variance. Most item-to-item correlations and all item-to-total correlations were moderate to strong (Spearman's rank correlation coefficient $r \geq 0.30$ ).

Table 3 Confirmatory factor analysis

\begin{tabular}{|c|c|c|c|c|c|c|}
\hline FACIT-Fatigue & RMSEA $(90 \% \mathrm{CI})$ & SRMR & CFI & $\omega$ & $\omega \mathrm{H}$ & ECV \\
\hline \multicolumn{7}{|l|}{ Single factor modeling } \\
\hline Symptom subscale & $0.068(0.005-0.123)$ & 0.014 & 0.998 & 0.903 & & \\
\hline Impact subscale & $0.166(0.143-0.190)$ & 0.061 & 0.961 & 0.907 & & \\
\hline Total scale & $0.152(0.139-0.165)$ & 0.066 & 0.946 & 0.943 & & \\
\hline Bifactor modeling & $0.120(0.105-0.135)$ & 0.044 & 0.973 & 0.954 & 0.905 & 0.819 \\
\hline
\end{tabular}

Abbreviations: Cl confidence interval, CFI comparative fit index, ECV explained common variance, FACIT-Fatigue Functional Assessment of Chronic Illness TherapyFatigue scale, RMSEA root mean square error of approximation, SRMR standardized root mean square residual, $\omega$ omega, $\omega H$ omega hierarchical Standard cutoff values: RMSEA $<0.06 ;$ SRMR $<0.08 ; \mathrm{CFI}>0.95$ [17-20] 


\section{Construct validity}

The FACIT-Fatigue symptom subscale, impact subscale and total scale scores correlated strongly with the EORTC QLQ-C30 global health status, physical function, role function and fatigue scale scores, and the EQVAS score (all Spearman's $r \geq 0.50$ ), demonstrating convergent validity (Table 4). Weak correlations (Spearman's $r<0.30$ ) were observed between the FACITFatigue scales, and the EORTC QLQ-C30 insomnia, constipation and diarrhea scales, indicating that there was no relationship between fatigue and these symptoms, and supporting divergent validity (Table 3).

\section{Known-groups validity}

Figure 1 shows the number of patients and mean FACI T-Fatigue scores at baseline by known groups. Knowngroups validity of the FACIT-Fatigue scales was demonstrated by significant differences between groups defined by baseline ECOG Performance Status score, Hb level and constitutional symptoms (Fig. 1).

\section{Defining the severity cut-off score}

Substantial agreement was observed between clusters grouped by FACIT-Fatigue and EORTC QLQ-C30 scores and the severe fatigue population defined using a FACIT-Fatigue total score threshold of either 30 or 34 (Cohen's kappa coefficients of 0.76 and 0.67 , respectively).

\section{Discussion}

This work demonstrates the validity and reliability of the FACIT-Fatigue, a PRO instrument that assesses fatiguerelated symptoms and impacts, in patients with CLL and shows that it is fit for purpose in this population. Cognitive debriefing of the FACIT-Fatigue in patients with $1 \mathrm{~L}$ or R/R CLL, conducted as part of qualitative interviews, confirmed that the instrument items are easily

Table 4 Correlations to assess the convergent and divergent validity of the FACIT-Fatigue scales versus other measures

\begin{tabular}{|c|c|c|c|}
\hline \multirow[b]{2}{*}{ FACIT-Fatigue vs: } & \multicolumn{3}{|l|}{ Spearman's $r$} \\
\hline & Symptom subscale & Impact subscale & Global Fatigue scale \\
\hline \multicolumn{4}{|l|}{ EORTC QLQ-C30 } \\
\hline Global health status & 0.66 & 0.64 & 0.69 \\
\hline Physical functioning & 0.61 & 0.71 & 0.71 \\
\hline Role functioning & 0.56 & 0.67 & 0.66 \\
\hline Emotional functioning & 0.49 & 0.54 & 0.55 \\
\hline Cognitive functioning & 0.37 & 0.52 & 0.48 \\
\hline Social functioning & 0.47 & 0.57 & 0.55 \\
\hline Fatigue & -0.75 & -0.75 & -0.80 \\
\hline Nausea/vomiting & -0.33 & -0.33 & -0.35 \\
\hline Pain & -0.39 & -0.43 & -0.44 \\
\hline Dyspnea & -0.34 & -0.32 & -0.35 \\
\hline Insomnia & -0.28 & -0.26 & -0.28 \\
\hline Appetite loss & -0.39 & -0.42 & -0.44 \\
\hline Constipation & -0.13 & -0.20 & -0.18 \\
\hline Diarrhea & -0.18 & -0.16 & -0.17 \\
\hline Financial problems & -0.21 & -0.31 & -0.29 \\
\hline \multicolumn{4}{|l|}{ EQ-5D-5L } \\
\hline Mobility & -0.40 & -0.45 & -0.46 \\
\hline Self-care & -0.19 & -0.32 & -0.29 \\
\hline Usual activities & -0.48 & -0.61 & -0.59 \\
\hline Pain/discomfort & -0.39 & -0.38 & -0.41 \\
\hline Anxiety/depression & -0.44 & -0.47 & -0.49 \\
\hline EQ-VAS score & 0.61 & 0.60 & 0.64 \\
\hline
\end{tabular}

Correlations are assessed based on absolute values. Strong correlations: absolute $r_{\mathrm{s}} \geq 0.50$; moderate correlation: absolute $0.30 \leq r_{\mathrm{s}}<0.50$; weak correlations: absolute $0.10 \leq r_{\mathrm{s}}<0.30$

Abbreviations: EORTC QLQ-C30 European Organisation for Research and Treatment of Cancer Quality of Life Questionnaire-Core 30-questions, EQ-5D-5L 5-level, 5-dimension EuroQol questionnaire, EQ-VAS EuroQol global health visual analog scale, FACIT-Fatigue Functional Assessment of Chronic Illness Therapy-Fatigue scale, $r_{\mathrm{s}}$ Spearman's $r$ 


\section{a Symptom scale}

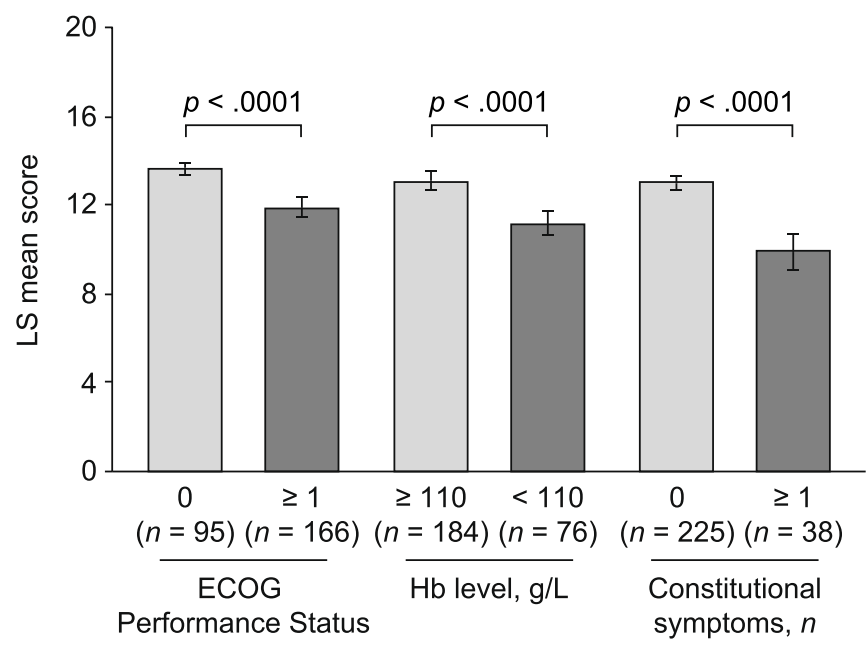

b Impact scale

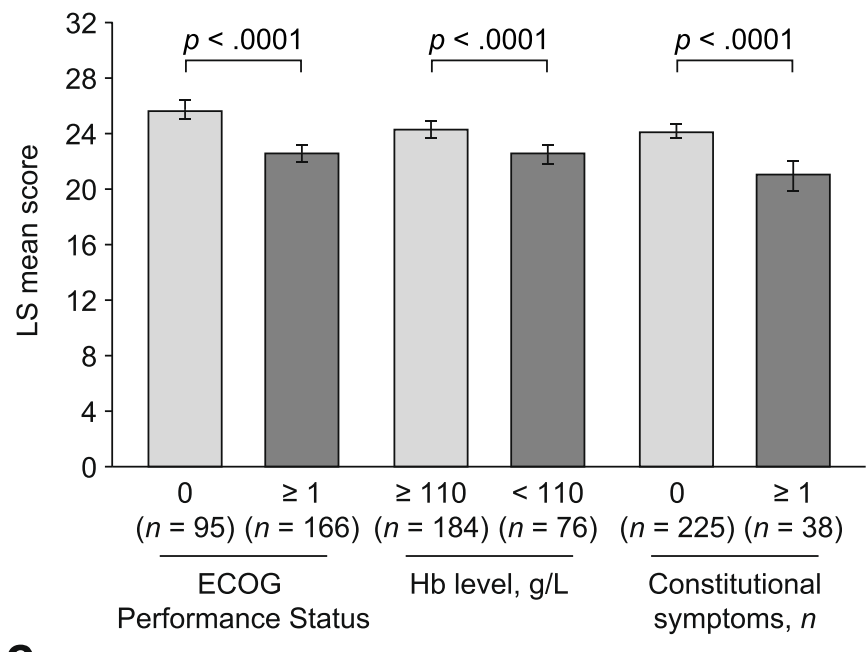

C Total scale

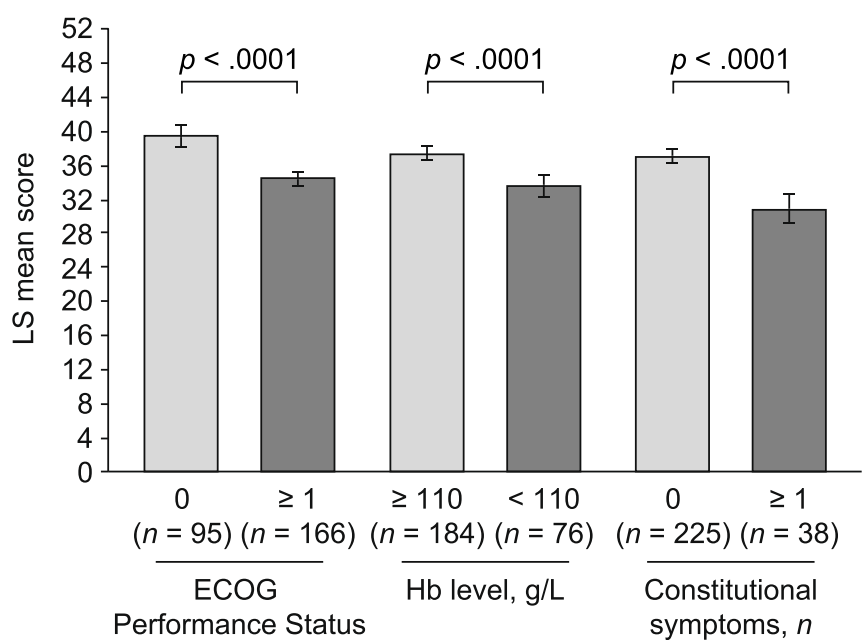

Fig. 1 (See legend on next page.) 
(See figure on previous page.)

Fig. 1 LS mean (SE) FACIT-Fatigue scores at baseline by known-groups. Information on ECOG Performance Status scores and Hb levels was unknown for two and three patients, respectively. Note that, because of the small sample size for ECOG Performance Status score 2, the known groups used here differ from the stratification of 0 or 1 vs 2 that were used as part of stratified randomization in the ASCEND trial. For two patients with ECOG Performance Status score $<2$, information was missing on whether their ECOG Performance Status score was 0 or 1. Abbreviations: ECOG Eastern Cooperative Oncology Group, FACIT-Fatigue Functional Assessment of Chronic Illness Therapy-Fatigue scale, Hb hemoglobin, LS least-squares, SE standard error

understood, interpreted as intended, relevant to patients with CLL at different disease stages and comprehensive. An exception was item 10, 'I am too tired to eat', which was not considered to be highly pertinent. The item is targeted to very severe fatigue, which often people cannot relate to unless they have experienced it themselves. This is consistent with findings from FACIT-Fatigue validation studies in other disease settings such as irondeficiency anemia, systemic lupus erythematosus and psoriatic arthritis [34-36]. However, patients thought that the item was still appropriate to retain.

The concept elicitation part of the qualitative interview study has been described previously [6]. It showed that fatigue is a key experience in patients with CLL that manifests as a variety of sub-components related to symptoms in CLL (tiredness/need for sleep; lack of energy; weakness; cognitive fatigue) and impacts (decreased ability to maintain social, familial or professional role; decreased physical functioning; frustration). In the current work, the FACIT-Fatigue items were shown to map well to the fatigue-related sub-components identified previously during concept elicitation, providing further support of the relevance and comprehensiveness of the FACIT-Fatigue in the CLL setting. Mean FACIT-Fatigue scores for patients with CLL were lower (worse) than general population scores $[5,13]$, indicating greater fatigue in the CLL population than the general population and providing further evidence that fatigue is a core component of CLL.

In the psychometric evaluation of phase 3 study data in patients with R/R CLL, CFA analysis and the examination of psychometrically informative bifactor-derived statistics supported the unidimensionality of the FACITFatigue. More specifically, the calculation of $\omega$ and $\omega \mathrm{H}$ indicated that a strong percentage of total score variance is attributable to the single general factor. As a consequence, we conclude that raw scores can essentially be assumed as indicators of the FACIT-Fatigue general factor and are not affected by the multidimensionality of the two subscales. The symptom and impact subscales could be distinguished as separate components that can be reported separately or combined as a total score, confirming previous analyses of the dimensionality of the FACIT-Fatigue [7]. In addition, our factor analysis findings, including strength of loadings and fit statistics, are consistent with the study of Cella et al. 2011 [7]. All three scales (symptom, impact, total) demonstrated good internal consistency, reliability, construct validity and known-groups validity, providing choice depending on purpose of use. The three FACIT-Fatigue scales differentiated between groups defined by disease severity indicators. Patients who were fully active without restrictions (ECOG Performance Status score 0) scored significantly better (i.e. less fatigue) on all scales than patients who were ambulatory but restricted in physically strenuous activity or unable to carry out any work activities (ECOG Performance Status score 1 or 2). Similarly, patients with no anemia or mild anemia $(\mathrm{Hb} \geq 110 \mathrm{~g} / \mathrm{L})$ scored significantly better on all three scales than those with moderate or severe anemia $(\mathrm{Hb}<110 \mathrm{~g} / \mathrm{L})$, and patients with no constitutional CLL symptoms scored significantly better than those with at least one such symptom.

In addition to demonstrating known-groups validity, the ability of the three FACIT-Fatigue scales to differentiate between groups based on disease severity indicators supports their relevance for use in clinical trials. Cluster analysis supported the previously identified FACIT-Fatigue total score thresholds of 30 and 34 to define a severe fatigue population.

A limitation of our work is that test-retest reliability of the FACIT-Fatigue was not assessed, because there was only one assessment time point (at baseline) before study drug initiation and no acceptable independent measures to identify patients remaining in a stable condition were used during the study. Interviewed patients were younger than those in the ASCEND trial (median age 58 years vs 67 years). Psychometric properties were evaluated in patients with R/R CLL only. Patients enrolled in a pivotal phase 3 CLL treatment trial may have less comorbidity than patients treated for CLL in the real world. Additional studies are needed to determine the instrument's responsiveness to change [20].

This work had important strengths, including the rigorous methodologies used, the comprehensive assessments conducted, an appropriate sample size for cognitive debriefing and the large sample size available for the psychometric evaluation.

\section{Conclusions}

Content validity assessment in patients with $1 \mathrm{~L}$ or $\mathrm{R} / \mathrm{R}$ CLL and psychometric evaluation in patients with $R / R$ 
CLL demonstrated that the FACIT-Fatigue has good psychometric properties and is fit for purpose in CLL. The three scoring options for the FACIT-Fatigue (symptom, impact, total) have similar reliability and validity, enabling choice depending on purpose of use. Results support the use of the FACIT-Fatigue in patients with $1 \mathrm{~L}$ or $\mathrm{R} / \mathrm{R}$ CLL in the clinical trial setting.

\section{Supplementary Information}

The online version contains supplementary material available at https:/doi. org/10.1186/s41687-021-00294-1.

Additional file 1 : Supplemental File 1. Proportion of patients with response 'not at all' and 'very much' for FACIT-Fatigue items.

Additional file $\mathbf{2}$ : Supplemental File $\mathbf{2}$. Factor loadings for the one factor models of the FACIT-Fatigue scale, Impact subscale and Symptom subscale.

Additional file 3 : Supplemental File 3. Factor loadings for the bifactor model of the FACIT-Fatigue.

\section{Abbreviations}

$1 \mathrm{~L}$ : First-line; CFA: Confirmatory factor analysis; CFI: Comparative fit index; Cl: Confidence interval; CLL: Chronic lymphocytic leukemia; ECOG: Eastern Cooperative Oncology Group; EORTC QLQ-30: European Organisation for Research and Treatment of Cancer Quality of Life Questionnaire-Core 30questions; EQ-5D-5L: 5-level 5-dimension Euro Qol questionnaire; EQVAS: EuroQol visual analogue scale; FACIT-Fatigue: Functional Assessment of Chronic Illness Therapy-Fatigue scale; Hb: Hemoglobin; LS: Least-squares; PRO: Patient-reported outcome; R/R: Relapsed or refractory; RMSEA: Root mean square error of approximation; SD: Standard deviation; SE: Standard error; SRMR: Standardized root mean square residual

\section{Acknowledgments}

Medical writing support was provided by Anja Becher, PhD, of Oxford PharmaGenesis, Oxford, UK, and was funded by AstraZeneca. We thank Christina Daskalopoulou, PhD, of IQVIA, Greece, for her help with performing the statistical analyses.

\section{Authors' contributions}

DE and DC conceptualized and designed the study. CI, LC, OM and DC collected the data. CI, LC, OM and DC analyzed the data. DE, CI, LC, OM and DC interpreted the results and substantively revised the manuscript. All authors approved the final version and are accountable for all aspects of the work.

\section{Funding}

This qualitative study was funded by AstraZeneca.

\section{Availability of data and materials}

Acerta Pharma, a member of the AstraZeneca Group, is committed to data transparency and will consider data sharing requests on a case-by-case basis. Any requests for de-identified patient data can be submitted to Acerta Pharma 3 months post-publication and ending 5 years following article publication with the intent-to-achieve aims of the original proposal. In addition, Acerta Pharma will provide the study protocol, statistical analysis plan, and informed consent form, as well as post results on clinicaltrials.gov, as required.

\section{Ethics approval and consent to participate}

The patient interviews were approved by the New England Institutional Review Board and all interviewed patients completed an informed consent form. The ASCEND trial protocol was approved by the institutional review board or independent ethics committee at each site, the study was conducted according to the principles of the Declaration of Helsinki and the International Conference on Harmonization Guidelines for Good Clinical Practice, and all patients provided written informed consent.
Consent for publication

Not applicable.

\section{Competing interests}

DE is an employee of AstraZeneca and holds shares in AstraZeneca. Cl, LC and OM are employees of IQVIA, which received funds from AstraZeneca to conduct the psychometric analysis of the study data. DC owns the FACIT questionnaires, and all related subscales, translations and adaptations (FACIT.org).

\section{Author details}

${ }^{1}$ AstraZeneca Gothenburg, Pepparedsleden 1, 43150 Mölndal, Sweden. ${ }^{2}$ IQVIA, Amsterdam, The Netherlands. ${ }^{3}$ IQVIA, Barcelona, Spain. ${ }^{4}$ IQVIA, New York, NY, USA. ${ }^{5}$ Northwestern University, Evanston, IL, USA.

Received: 10 August 2020 Accepted: 2 February 2021

Published online: 11 March 2021

\section{References}

1. Hallek, M. (2017). Chronic lymphocytic leukemia: 2017 update on diagnosis, risk stratification, and treatment. American Journal of Hematology, 92, 946965.

2. Hallek, M., Cheson, B. D., Catovsky, D., Caligaris-Cappio, F., Dighiero, G., Dohner, H., ... Kipps, T. J. (2018). iwCLL guidelines for diagnosis, indications for treatment, response assessment, and supportive management of CLL. Blood, 131, 2745-2760

3. Bower, J. E. (2014). Cancer-related fatigue - Mechanisms, risk factors, and treatments. Nature Reviews. Clinical Oncology, 11, 597-609.

4. World Health Organization (2011). Haemoglobin concentrations for the diagnosis of anaemia and assessment of severity. Vitamin and Mineral Nutrition Information System. Geneva: World Health Organization (WHO/ NMH/NHD/MNM/11.1) Available at: http://www.who.int/vmnis/indicators/ha emoglobin.pdf. Accessed 6 Nov 2020.

5. Cella, D., Lai, J. S., Chang, C. H., Peterman, A., \& Slavin, M. (2002). Fatique in cancer patients compared with fatigue in the general United States population. Cancer, 94, 528-538.

6. Eek, D., Blowfield, M., Krogh, C., Chung, H., \& Eyre, T. A. (2020). Development of a conceptual model of chronic lymphocytic leukemia to better understand the patient experience. Patient [ePub ahead of print].

7. Cella, D., Lai, J. S., \& Stone, A. (2011). Self-reported fatigue: one dimension or more? Lessons from the functional assessment of chronic illness therapyfatigue (FACIT-F) questionnaire. Support Care Cancer, 19, 1441-1450.

8. Butt, Z., Lai, J. S., Rao, D., Heinemann, A. W., Bill, A., \& Cella, D. (2013) Measurement of fatigue in cancer, stroke, and HIV using the functional assessment of chronic illness therapy - fatigue (FACIT-F) scale. Journal of Psychosomatic Research, 74, 64-68.

9. Smith, E., Lai, J. S., \& Cella, D. (2010). Building a measure of fatigue: The functional assessment of chronic illness therapy fatigue scale. $P M \& R$ : The Journal of Injury, Function, and Rehabilitation, 2, 359-363.

10. Lai, J. S., Cook, K., Stone, A., Beaumont, J., \& Cella, D. (2009). Classical test theory and item response theory/Rasch model to assess differences between patient-reported fatigue using 7-day and 4-week recall periods. Journal of Clinical Epidemiology, 62, 991-997.

11. Efficace, F., Cottone, F., Oswald, L. B., Cella, D., Patriarca, A., Niscola, P., ... Vignetti, M. (2020). The IPSS-R more accurately captures fatigue severity of newly diagnosed patients with myelodysplastic syndromes compared with the IPSS index. Leukemia, 34, 2451-2459.

12. Ghia, P., Pluta, A., Wach, M., Lysak, D., Kozak, T., Simkovic, M., ... Jurczak, W. (2020). ASCEND: Phase III, randomized trial of acalabrutinib versus idelalisib plus rituximab or bendamustine plus rituximab in relapsed or refractory chronic lymphocytic leukemia. Journal of Clinical Oncology, 38(25), 28492861

13. Montan, I., Lowe, B., Cella, D., Mehnert, A., \& Hinz, A. (2018). General population norms for the functional assessment of chronic illness therapy (FACIT)-fatigue scale. Value in Health, 21, 1313-1321.

14. Bland, J. M., \& Altman, D. G. (1997). Cronbach's alpha. BMJ, 314, 572

15. Reise, S. P., Scheines, R., Widaman, K. F., \& Haviland, M. G. (2013). Multidimensionality and structural coefficient bias in structural equation modeling: A bifactor perspective. Educational and Psychological Measurement, 73, 5-26. 
16. Rodriguez, A., Reise, S. P., \& Haviland, M. G. (2016). Applying bifactor statistical indices in the evaluation of psychological measures. Journal of Personality Assessment, 98, 223-237.

17. Browne, M. W., \& Cudeck, R. (1993). In K. A. Bollen, \& J. S. Long (Eds.) Alternative ways of assessing model fit. In: Testing structural equation models. Newbury Park: Sage Publications.

18. Bentler, P. M. (1995). EQS structural equations program manual. Encino: Multivariate Software.

19. Bentler, P. M. (1990). Comparative fit indexes in structural models. Psychological Bulletin, 107, 238-246.

20. Cook, K. F., Kallen, M. A., \& Amtmann, D. (2009). Having a fit: Impact of number of items and distribution of data on traditional criteria for assessing IRT's unidimensionality assumption. Quality of Life Research, 18, 447-460.

21. Muthén, L. K., \& Muthén, B. O. (1998-2017). Mplus User's guide, (8th ed., ). Los Angeles: Muthén \& Muthén.

22. Asparouhoy, T., \& Muthén, B. (2010). Weighted least squares estimation with missing data. Mplus technical appendix. Los Angeles: Muthén \& Muthén.

23. Fayers, P. M., \& Machin, D. (2007). Quality of life: The assessment, analysis and interpretation of patient-reported outcomes, (2nd ed., ). New York: Wiley.

24. Dunn, T. J., Baguley, T., \& Brunsden, V. (2014). From alpha to omega: A practical solution to the pervasive problem of internal consistency estimation. British Journal of Psychology, 105, 399-412.

25. Zinbarg, R. E., Revelle, W., Yovel, I., \& Li, W. (2005). Cronbach's a, Revelle's $\beta$ and McDonald's $\omega \mathrm{H}$ : Their relations with each other and two alternative conceptualizations of reliability. Psychometrika, 70, 123-133.

26. Zinbarg, R. E., Yovel, I., Revelle, W., \& McDonald, R. P. (2006). Estimating generalizability to a latent variable common to all of a Scale's indicators: A comparison of estimators for wh. Applied Psychological Measurement, 30, $121-144$.

27. Reise, S. P., Moore, T. M., \& Haviland, M. G. (2010). Bifactor models and rotations: Exploring the extent to which multidimensional data yield univocal scale scores. Journal of Personality Assessment, 92, 544-559.

28. Ten Berge, J. M. F., \& Sočan, G. (2004). The greatest lower bound to the reliability of a test and the hypothesis of unidimensionality. Psychometrika 69, 613-625.

29. Cohen, J. (1992). A power primer. Psychological Bulletin, 112, 155-159.

30. Everitt, B. S., Landau, S., \& Leese, M. (2001). Cluster analysis, (4th ed., ). London: Arnold

31. IBM Corp. Released (2015). IBM SPSS statistics for windows, version 23.0. Armonk: IBM Corp.

32. Piper, B. F., \& Cella, D. (2010). Cancer-related fatigue: Definitions and clinical subtypes. Journal of the National Comprehensive Cancer Network, 8, 958-966.

33. Cohen, J. (1960). A coefficient of agreement for nominal scales. Educational and Psychological Measurement, 20, 37-46.

34. Cella, D., Wilson, H., Shalhoub, H., Revicki, D. A., Cappelleri, J. C., Bushmakin, A. G., ... Hsu, M. A. (2019). Content validity and psychometric evaluation of functional assessment of chronic illness therapy-fatigue in patients with psoriatic arthritis. Journal of Patient-Reported Outcomes, 3, 30.

35. Kosinski, M., Gajria, K., Fernandes, A. W., \& Cella, D. (2013). Qualitative validation of the FACIT-fatigue scale in systemic lupus erythematosus. Lupus, $22,422-430$

36. Acaster, S., Dickerhoof, R., DeBusk, K., Bernard, K., Strauss, W., \& Allen, L. F. (2015). Qualitative and quantitative validation of the FACIT-fatigue scale in iron deficiency anemia. Health and Quality of Life Outcomes, 13, 60.

\section{Publisher's Note}

Springer Nature remains neutral with regard to jurisdictional claims in published maps and institutional affiliations.

\section{Submit your manuscript to a SpringerOpen ${ }^{\circ}$ journal and benefit from:}

- Convenient online submission

- Rigorous peer review

- Open access: articles freely available online

- High visibility within the field

- Retaining the copyright to your article

Submit your next manuscript at $\boldsymbol{\nabla}$ springeropen.com 